the 16th of December. My friend Dr. Macintyre threw out the idea that, after all, the case may have been one of neuralgia; and he supported his opinion by some ingenious, though $\mathbf{I}$ did not think conclusive arguments. The exfoliation of bone at an early part of the history of the case, and the swelling, heat, and redness, which have always attended the violent attacks of pain, seemed to me to militate much against such an idea. Notwithstanding this consideration, however, as an issue was open, and as I, in common with the rest of the profession, entertain a very high respect for Dr. Macintyre's experience and judgment, I acted upon it so far as to give my patient the benefit of the endermic use of morphine, which I had seen, in my own practice, to be very serviceable in long-standing neuralgia of the leg. One patient, more particularly, was cured by me, some years ago, of an obstinate and most severe neuralgia of one of his legs by this method, and I did not think myself justified in omitting it in the present instance, though the two cases had nothing in common except the violent pain, and though it was proceeding on a principle somewhat different from, but by no means contrary to, that on which the other parts of the treatment had been conducted. Some time after this, Dr. Macintyre was invited to visit the patient, and he entirely approved of my proceedings, and was of opinion that a cure had been effected.

April 18th.- This morning Mr. M- left London for Scotland in good health. His leg is perfectly well, giving him no pain or uneasiness.

I have purposely delayed publishing this interesting and gratifying case till now, that it might be seen how far time would sanction the hopes entertained of a permanent cure. So far as three months' residence in the Highlands, together with much fatigue undergone during that time, can give such a sanction, the result is in the highest degree satisfactory. Mr. M- remains perfectly well. I conclude with one other remark. If my diagnosis was correct in this case, - and the probability is that it was so, both from the symptoms and from the high authority of Sir Benjamin Brodie,-and if a permanent cure has been accomplished, then another triumph has been added to the many which a long and patiently continued course of the iodide of potassium has achieved over many of the worst and most obstinate forms of disease; and this is of no mean consequence, since the only alternative in the case before us was a painful surgical operation.

Harley-street, July 24, 1844 .

\section{ON THE TREATMEN'T OF HYDROCELE.}

By J. Adams, Esq. Assistant-Surgeon to the London Hospital.

I wISH, through the medium of THE LANCET, to recommend for trial a very simple method of treating hydrocele, with the view to effect a radical cure. I have tried it in one instance, and the success is such as to warrant an extended trial of its efficacy. It has the advantages of great simplicity and entire freedom from danger. The case in which I applied it was one of hydrocele, after the operation by incision had but partially effected a cure; and some time after the operation, a collection of fluid occurred at the back part of the testis, the gland being pushed forward by the irregular distention of the sac, in consequence of the partial obliteration of the cavity. Having drawn off the fluid with a moderate-sized trocar, I introduced through the canula a camel's bair brush, dipped in a strong tincture of iodine, and carefully painted the interior of the sac with this fluid. It was succeeded by the ordinary train of symptoms, and at the present time there is every indication of complete success. The operation was performed about seven weeks since.

31, New Broad Street, Oct. 24, 1844.

\section{ACCIDENTAL POISONING WITH ARSENIC,} SUCCESSFULLY

TREATED WITH THE HYDRATED PER-OXIDE OF IRON.

By J. J. KeLso, M.D. Lisburn.

Trodgr a wide-spread scepticism regarding the efficacy of the hydrated peroxide or sesquioxide of iron, as an antidote to poisoning with arsenic, most undoubtedly, and-considering the number of different substances that have successively been proposed for this purpose, only to be rejected each in turn as utterly valueless-notunnaturally, obtains; still, from repeated experiments, both of a physiological and chemical nature, and more especially from actual experience of the preparation in cases of poisoning in the human subject, its power of converting the white oxide of this metal into an insoluble compound-the arsenite of iron-and thereby rendering it innoxious in its effects, as well in man as in the lower animals, seems at present to be pretty well established, and very generally admitted by many members of the profession -and, among others, by Christison* and Pereira, fon whose authority we are accustomed, not without sufficient reason, to repose. It may not, perhaps, be too much to affirm, that on the whole a growing faith in favour of the counteracting influence of the rust of iron, either in the state of magna or dry powder, in instances where arsenic has been ingested in poisonous doses, at least under certain conditions, and to a certain extent, is evidently and very reasonably beginning to manifest itself. Be this as it may, the following case presents, I am strongly inclined to think, a very striking exemplification of the good effects of the remedy.

Mary C_- a fine, healthy, intelligent little girl, of about eight years of age, swallowed, on the 18th of May last, a quantity of arsenic-or at least what then was believed to be such-which had been designedly mixed with some oatmeal, and set aside in a particular place, for the purpose of poisoning rats.

The facts of this very interesting case are briefly as follow:$\mathrm{C}$ - the child's father, it appeared, purchased on the preceding evening, at an apothecary's shop in Lisburn, a pennyworth of white arsenic, in powder, which I have every reason to believe might have amounted in quantity to upwards of an ounce at least. This, indeed, is admitted by the gentleman himself who vended the article, and whose recollection of the transaction, when questioned on the subject, enabled him besides to verify the still more important fact of the substance itself being arsenic - the white oxide, of course, of the shops. On reaching home, he took of the substance thus procured, as near as he could estimate, rather more than the one-half, and having mixed it with more than double its bulk of dry oatmeal, he distributed it equally on two pieces of broken crockery, which he placed, at bedtime, in separate places, in an apartment of the house, for the purpose of destroying the rats, with which it is, it appears, infested. The operation, he says, was performed in the presence of his wife and the girl in question, in common with two or three others of the more advanced members of the family. He further particularly states his having, at the time, distinctly and repeatedly mentioned in the presence-and hearing, of course - of the family group, who were closely congregated in witnessing the interesting procedure around him, the fact of the powder which he was commixing being very poisonous in its character, and that its object was for the destruction of rats. However that may be, his wife, in the morning, took up, for the sake of greater security, both pieces of crockery containing the mixture indicated, or rather, the portions that remained unconsumed by the vermin, which she says was considerable. One of these she very properly placed in a spot beyond the possibility of reach by any of the children, while the other, quite unaccountably, she left in a place that was easily accessible by almost all. The consequence was, that not long after, our little patient, in her rambles, came upon the dish containing the residue of the poisonous mixture, and, in forgetfulness or ignorance of what it really was, at once consumed the entire contents.

This occurred at about eleven o'clock A.M., and in rather less than an hour and a half after, I saw her. Her mother, who seemed in great distress, informed me that the first intimation she had of the startling fact of the child having eaten the poisonous compound, was its commencing to vomit on being presented with some food. She immediately had recourse, she further stated, to the popular remedy of melted butter, which she administered in considerable quantity. This had the effect of exciting rather free vomiting, which had been repeated, it appears, two or three times Her symptoms then were these:-Marked listlessness and indisposition to reply to questions; pulse about 120, weak, and rather unequal, with occasional indications of fluttering; respiration not irregular, but rather slow, and imperfectly performed; and a feeling of sickness or nausea, with an inclination every now and then to vomit. When asked if she had any feeling of heat or pain in the throat or stomach, she gave me distinctly to understand that she experienced, in some degree, both of these sensations. The pain in the epigastric region was evidently increased on the application of the hand, as she then became fearful, and complained of the procedure. The pupil of either eye, but especially the right one, was rather remarkably, and, in cases of this kind, I believe, unusually, influenced. Alternate dilatations and contractions, in rather rapid and extreme degree, were observed to occur; these were evidently still further increased on the presentation of a lighted candle, or the tip of the forefinger motioning to and fro. There was no other derangement of function deserving mention; vital, physical, or mental. 